\title{
Color Octet Electron Search Potential of the FCC Based e-p Colliders
}

\author{
Y. C. Acar \\ Department of Electrical and Electronics Engineering, \\ TOBB University of Economics and Technology, Ankara, Turkey" \\ U. Kaya \\ Department of Material Science and Nanotechnology, \\ TOBB University of Economics and Technology, Ankara, Turkey and \\ Department of Physics, Faculty of Sciences, Ankara University, Ankara, Turkey \\ B. B. Oner \\ Department of Material Science and Nanotechnology, \\ TOBB University of Economics and Technology, Ankara, Turkey \\ S. Sultansoy \\ Department of Material Science and Nanotechnology, \\ TOBB University of Economics and Technology, Ankara, Turkey and \\ ANAS Instute of Physics, Baku, Azerbaijan
}

Resonant production of color octet electron, $e_{8}$, at the FCC based ep colliders has been analyzed. It is shown that e-FCC will cover much a wider region of $e_{8}$ masses compared to the LHC. Moreover, with highest electron beam energy, $e_{8}$ search potential of the e-FCC exceeds that of FCC pp collider. If $e_{8}$ is discovered earlier by the FCC pp collider, e-FCC will give opportunity to handle very important additional information. For example, compositeness scale can be probed up to hundreds $\mathrm{TeV}$ region.

\footnotetext{
* ycacar@etu.edu.tr

$\dagger$ ukaya@etu.edu.tr

$\ddagger$ ssultansoy@etu.edu.tr
} 


\section{INTRODUCTION}

Standard Model (SM) has proven its reliability by the experimental verifications of its particle content in the recent decades. SM puzzle has been completed by the discovery of Higgs boson [1, 2, However, SM seems not to be the end of the whole story. There are still many unsolved problems that are out of the scope of the SM and especially the large number of currently known elementary particles becomes more of an issue. For this reason a lot of BSM models have been proposed including extension of scalar and fermionic sectors of SM, enlargement of SM gauge symmetry group, SUSY, compositeness (preons [3]), extra dimensions etc. Keeping in mind historical development of fundamental building blocks of matter, the search for preonic models seem to be quite natural. This development is summarized in Table I.

Table I. Historical development of fundamentality.

\begin{tabular}{|r|c|c|c|}
\hline Stages & $1870-1930 \mathrm{~s}$ & $1950-1970 \mathrm{~s}$ & $1970-2030 \mathrm{~s}$ \\
\hline \hline Fundamental Constituent Inflation & Chemical Elements & Hadrons & Quarks, Leptons \\
\hline Systematics & Periodic Table & Eight-fold way & Family Replication \\
\hline Confirmed Predictions & New Elements & New Hadrons & BSM particles \\
\hline Clarifying Experiments & Rutherford & SLAC-DIS & LHC or rather FCC? \\
\hline Building Blocks & Proton, Neutron, Electron & Quarks & Preons? \\
\hline Energy Scale & $\mathrm{MeV}$ & GeV & TeV? \\
\hline Impact on Technology & Exceptional & Indirect & Exceptional \\
\hline
\end{tabular}

Family replication and especially SM fermion mixings can be considered as indications of preonic structure of matter. One of the notable results of preonic models is prediction of well-known BSM particles (such as excited leptons and quarks, leptoquarks) and contact interactions which are widely investigated by ATLAS and CMS. In composite models with colored preons (see [4] and references therein), leptons have color octet partners, $\ell_{8}$, which are known as leptogluons. Phenomenologically their status is similar to excited leptons and leptoquarks. Experimentally excited leptons and leptoquarks are considered in CMS and ATLAS experiment searches, however, there is no direct search on leptogluons.

There are a number of phenomenological studies on $\ell_{8}$ production at $\mathrm{TeV}$ colliders. For example, production of leptogluons at the LHC has been analyzed in [5-9]. Resonant production of leptogluons at ep and $\mu$ p colliders were considered in 10 12 and [13, respectively. Indirect production of leptogluons at ILC and CLIC has been studied in [14. On the other hand, considering IceCube PeV events [15], color octet neutrinos may be source of these extraordinary events [16].

Experimental bound on color octet electron $\left(e_{8}\right), M_{e_{8}}>86 \mathrm{GeV}$, presented in [17] is based on 25 years old CDF search for pair production of unit-charged particles which leave the detector before decaying [18]. As mentioned in 19] DO clearly excluded $200 \mathrm{GeV}$ leptogluons decaying within the detector. The twenty years old H1 search for $e_{8}$ has excluded the compositeness scale $\Lambda<3 \mathrm{TeV}$ for $M_{e_{8}} \approx 100 \mathrm{GeV}$ and $\Lambda<240 \mathrm{GeV}$ for $M_{e_{8}} \approx 250 \mathrm{GeV}[20$, 21 . While the LEP experiments did not perform dedicated search for leptogluons, low limits for excited lepton masses, namely $103.2 \mathrm{GeV}$ [17], certainly is valid for $\ell_{8}$, too. Finally, reconsideration of CMS results on leptoquark searches performed in [7] leads to the strongest current limit on the $e_{8}$ mass, $M_{e_{8}}>1.2-1.3 \mathrm{TeV}$.

The advantage of ep colliders with sufficiently high center of mass $(\mathrm{CM})$ energies is that $e_{8}$ is produced in resonance mode. Large Hadron electron Collider [22] (LHeC) is the highest center of mass energy ep collider proposal up to date. Unfortunately, approved option which assumes $60 \mathrm{GeV}$ energy recovery linac for electron beam [23, will not give an opportunity to cover $e_{8}$ masses above $1.3 \mathrm{TeV}$ [11]. For this reason, ep colliders with higher energies should be considered for resonant production of $e_{8}$.

In this paper, we consider resonant production of $e_{8}$ at the FCC based ep colliders. Main parameters of these colliders are given in Section II. Phenomenology of $e_{8}$ is presented in Section III. In Section IV, signal and background analyses have been performed and discovery limits on $e_{8}$ mass are estimated. Compositeness scale matters are discussed in Section V. Finally we summarize our results in Section VI.

\section{FCC BASED $e p$ COLLIDERS}

It is widely known that lepton-hadron collisions have been playing a crucial role in exploration of deep structure of matter. For example, electron scattering on atomic nuclei reveals structure of nucleons in Hofstadter experiment [24, quark parton model was originated from lepton-hadron collisions etc [25]. Investigation of extremely small $x$ 
but sufficiently high $Q^{2}$ will provide a basis for deeper understanding of the nature of strong interactions at all levels ranging from nucleus to partons. In addition, the results from lepton-hadron colliders are necessary for adequate interpretation of physics at possible future hadron colliders. Today, linac-ring type ep machines seem to be the most convenient way to $\mathrm{TeV}$ scale in lepton-hadron collisions; and it is also possible that in future, $\mu \mathrm{p}$ machines can also be considered depending on solutions of the principal issues of the $\mu^{+} \mu^{-}$colliders.

FCC [26] is future $100 \mathrm{TeV}$ CM energy pp collider proposed at CERN and supported by European Union within the Horizon 2020 Framework Programme for Research and Innovation. It includes also an electron-positron collider option at the same tunnel (TLEP), as well as ep collider options. Construction of future $e^{+} e^{-}$colliders and $\mu^{+} \mu^{-}$ colliders tangential to FCC will give opportunity to achieve highest $\mathrm{CM}$ energies in ep and $\mu \mathrm{p}$ collisions [27 29. A possible configuration of FCC based lepton-hadron colliders is shown in Figure 1.

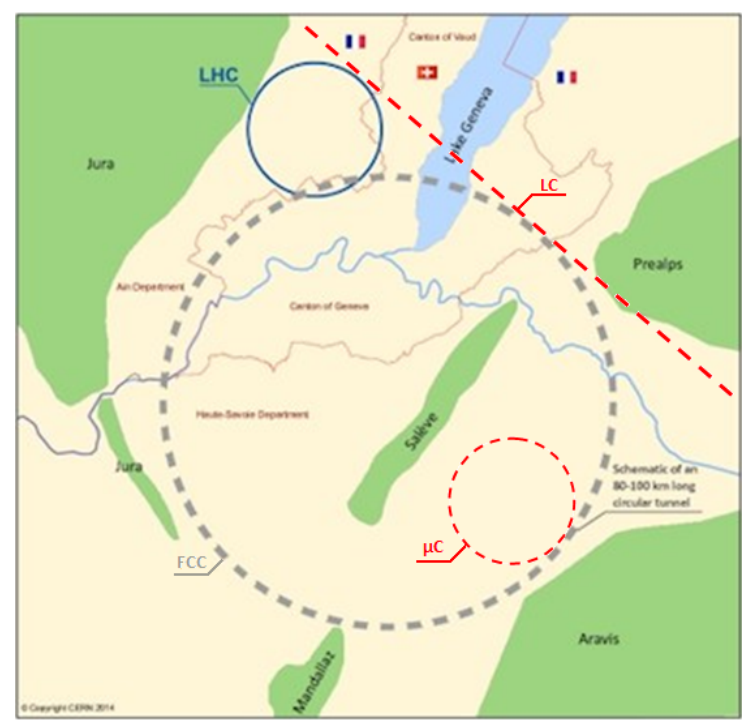

Figure 1. Possible configuration of FCC, linear collider (LC) and muon collider $(\mu \mathrm{C})$.

CM energy and luminosity values for FCC based ep colliders - with three different options of electron beam energy - which we use in this study are given in Table II. In this table ERL60 denotes energy recovery linac proposed for LHeC, ILC means International Linear Collider [30] with highest energy and PWFA-LC denotes Plasma Wake-Field Accelerator Linear Collider 31] with highest energy (for details see refs. 27 29]). In principle, staged scheme can be considered for the FCC based ep colliders: starting from ERL60 $\otimes \mathrm{FCC}$, through ILC $\otimes \mathrm{FCC}$ to the highest CM energy PWFA-LC $\otimes$ FCC.

Table II. Main parameters of the FCC based ep colliders.

\begin{tabular}{|c|c|c|c|}
\hline Collider Name & $E_{e}, \mathrm{TeV}$ & $\mathrm{CM}$ Energy, TeV & $L_{i n t}, \mathrm{fb}^{-1}$ per year \\
\hline \hline ERL60 $\otimes \mathrm{FCC}$ & 0.06 & 3.46 & 100 \\
\hline ILC $\otimes \mathrm{FCC}$ & 0.5 & 10 & $10-100$ \\
\hline PWFA-LC $\otimes \mathrm{FCC}$ & 5 & 31.6 & $1-10$ \\
\hline
\end{tabular}

\section{COLOR OCTET ELECTRON}

In fermion-scalar models with colored preons, leptons are bound states of one fermionic color triplet preon and one scalar color triplet anti-preon

$$
\ell=(F \bar{S})=3 \otimes \overline{3}=1 \oplus 8
$$


therefore, each SM lepton has one color octet partner. In three-fermion models with color triplet fermionic preons the color decomposition is

$$
\ell=(F F F)=3 \otimes 3 \otimes 3=1 \oplus 8 \oplus 8 \oplus 10
$$

and each SM lepton has two color octet and one color decuplet partners. Concerning the relation between compositeness scale and masses of leptogluons, two scenarios can be considered: $M_{e_{8}} \approx \Lambda$ (QCD-like scenario) and $M_{e_{8}} \ll \Lambda$ (Higgs-like scenario). In the second scenario SM-like hierarchy may be realized, namely, $M_{e_{8}} \ll M_{\mu_{8}} \ll M_{\tau_{8}} \ll \Lambda$. Interaction lagrangian of $\ell_{8}$ with leptons and gluons can be written as [11, 17,

$$
L=\frac{1}{2 \Lambda} \sum_{l}\left\{\overline{\ell_{8}^{\alpha}} g_{s} G_{\mu \nu}^{\alpha} \sigma^{\mu \nu}\left(\eta_{L} \ell_{L}+\eta_{R} \ell_{R}\right)+h . c .\right\}
$$

where $g_{s}$ is strong coupling constant, $\Lambda$ denotes compositeness scale, $G_{\mu \nu}$ is gluon field strength tensor, $\ell_{L(R)}$ stands for left (right) spinor components of lepton, $\ell=e, \mu, \tau ; \sigma^{\mu \nu}$ is the antisymmetric tensor $\left(\sigma^{\mu \nu}=\frac{i}{2}\left[\gamma^{\mu}, \gamma^{\nu}\right]\right), \eta_{L}\left(\eta_{R}\right)$ symbolizes chirality factor. Keeping in mind leptonic chiral invariance $\left(\eta_{L} \eta_{R}=0\right)$, we take $\eta_{L}=1$ and $\eta_{R}=0$. Decay width of $\ell_{8}$ given by

$$
\Gamma\left(\ell_{8} \rightarrow \ell+g\right)=\frac{\alpha_{s} M_{\ell_{8}}^{3}}{4 \Lambda^{2}}
$$

where $\alpha_{s}=g_{s} / 4 \pi$. The decay width of $e_{8}$ is presented in Fig. 2 for $\Lambda=M_{e_{8}}$ and $\Lambda=100 \mathrm{TeV}$.

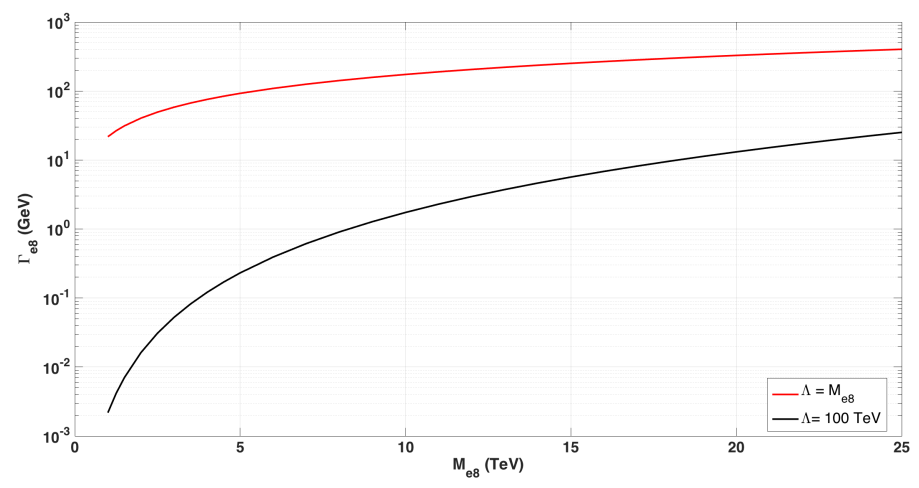

Figure 2. $e_{8}$ decay width vs its mass for $\Lambda=M_{e_{8}}$ and $\Lambda=100 \mathrm{TeV}$.

Diagram for resonant production of $e_{8}$ is shown in Figure 3. We implement model files of $e_{8}$ into MadGraph5 event generator 32] and use CTEQ6L1 parton distribution function [33] for numerical calculations. MadGraph5-Pythia6 interface was used for parton showering and hadronization [34].

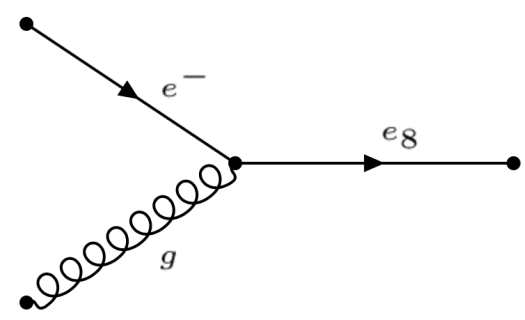

Figure 3. Feynman Diagram for resonant production of $e_{8}$ in ep collisions. 
The resonant $e_{8}$ production cross sections for different options of the FCC based ep colliders (Table II) are presented in Fig. 4 (for $\Lambda=M_{e_{8}}$ and $\Lambda=100 \mathrm{TeV}$ cases).
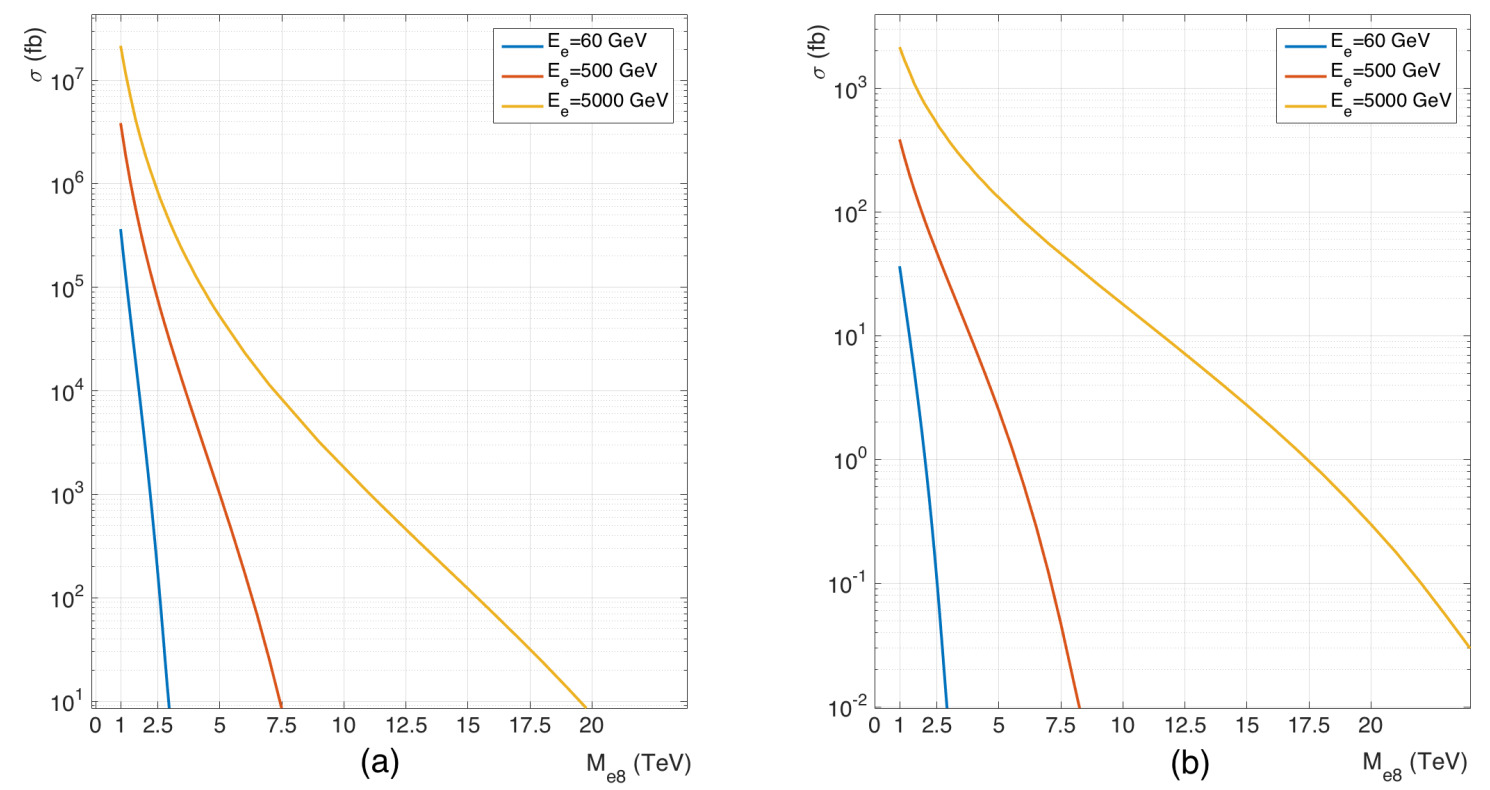

Figure 4. Resonant production of $e_{8}$ at the FCC based ep colliders for $\Lambda=M_{e_{8}}$ (a) and $\Lambda=100 \mathrm{TeV}$ (b).

In order to emphasize the advantage of the resonant production let us compare the production of $e_{8}$ with mass $10 \mathrm{TeV}$ at PWFA-LC $\otimes \mathrm{FCC}\left(\sqrt{s_{e p}}=31.6 \mathrm{TeV}, L_{i n t}=10 \mathrm{fb}^{-1}\right)$ and FCC-pp option $\left(\sqrt{s_{p p}}=100 \mathrm{TeV}, L_{i n t}=500\right.$ $\left.f b^{-1}\right)$. As seen from Fig. 4, production cross section at ep is $2000 \mathrm{fb}$ for $\Lambda=10 \mathrm{TeV}$ and $20 \mathrm{fb}$ for $\Lambda=100 \mathrm{TeV}$, whereas corresponding cross sections for pair production of $e_{8}$ at the FCC-pp are $\sim 0.50 f b$ and $0.29 f b$, respectively. Therefore, numbers of produced $e_{8}$ are $n=20000$ at ep and $n=250$ at pp if $\Lambda=10 \mathrm{TeV}$. Corresponding numbers for $\Lambda=100 \mathrm{TeV}$ are $n=200$ and $n=188$, respectively. Keeping in mind that ep collisions have more clear experimental environment than pp collisions, PWFA-LC $\otimes$ FCC seems to be more advantageous even for $\Lambda=100 \mathrm{TeV}$ case.

\section{SIGNAL - BACKGROUND ANALYSIS}

In this section numerical calculations will be performed for $\Lambda=M_{e_{8}}$. In order to determine appropriate kinematical cuts $p_{T}$ and $\eta$ distributions for signal and background processes are computed. At this stage, generic cuts on electron and jet transverse momentum are chosen as $p_{T_{e}}=20 \mathrm{GeV}$ and $p_{T_{j}}=30 \mathrm{GeV}$, respectively. Let us mention that jet corresponds to gluon for signal $\left(e g \rightarrow e_{8} \rightarrow e g\right.$ at partonic level) and quarks for main background (eq $\rightarrow e q$ through $\gamma$ and $Z$ exchanges) processes. Then discovery cuts on $p_{T}$ and $\eta$ are determined for different electron beam energy values and the invariant mass distributions are presented with these cuts. Finally, discovery limits on the color octet electron are presented.

Let us start with ERL60®FCC. Transverse momentum distribution of final state electrons (the same as for jets) is presented in Figure 5a. Keeping in mind that $M_{e_{8}}<1.3 \mathrm{TeV}$ is excluded by the reconsideration of CMS results on leptoquark search [6], discovery cut $p_{T}>500 \mathrm{GeV}$ seems to be adequate. Pseudorapidity distributions for electron and jets are shown in Figures $5 \mathrm{~b}$ and $5 \mathrm{c}$, respectively. As can be seen from Figure $5 \mathrm{~b}, \eta_{e}>0.5$ cut drastically reduces the background while keeping the signal almost unaffected. In similar manner, $\eta_{j}>2.1$ is chosen. Upper limit for both $\eta_{e}$ and $\eta_{j}$ is taken as $\eta_{e}, \eta_{j}<4.74$ which corresponds to $1^{o}$ in proton direction. This value can be covered by very forward detector as in the LHeC case [22]. Invariant mass distributions with generic cuts and discovery cuts are presented in Figures 5d and 6, respectively. It is seen that after discovery cuts, background goes down essentially below signal in the invariant mass distribution. 

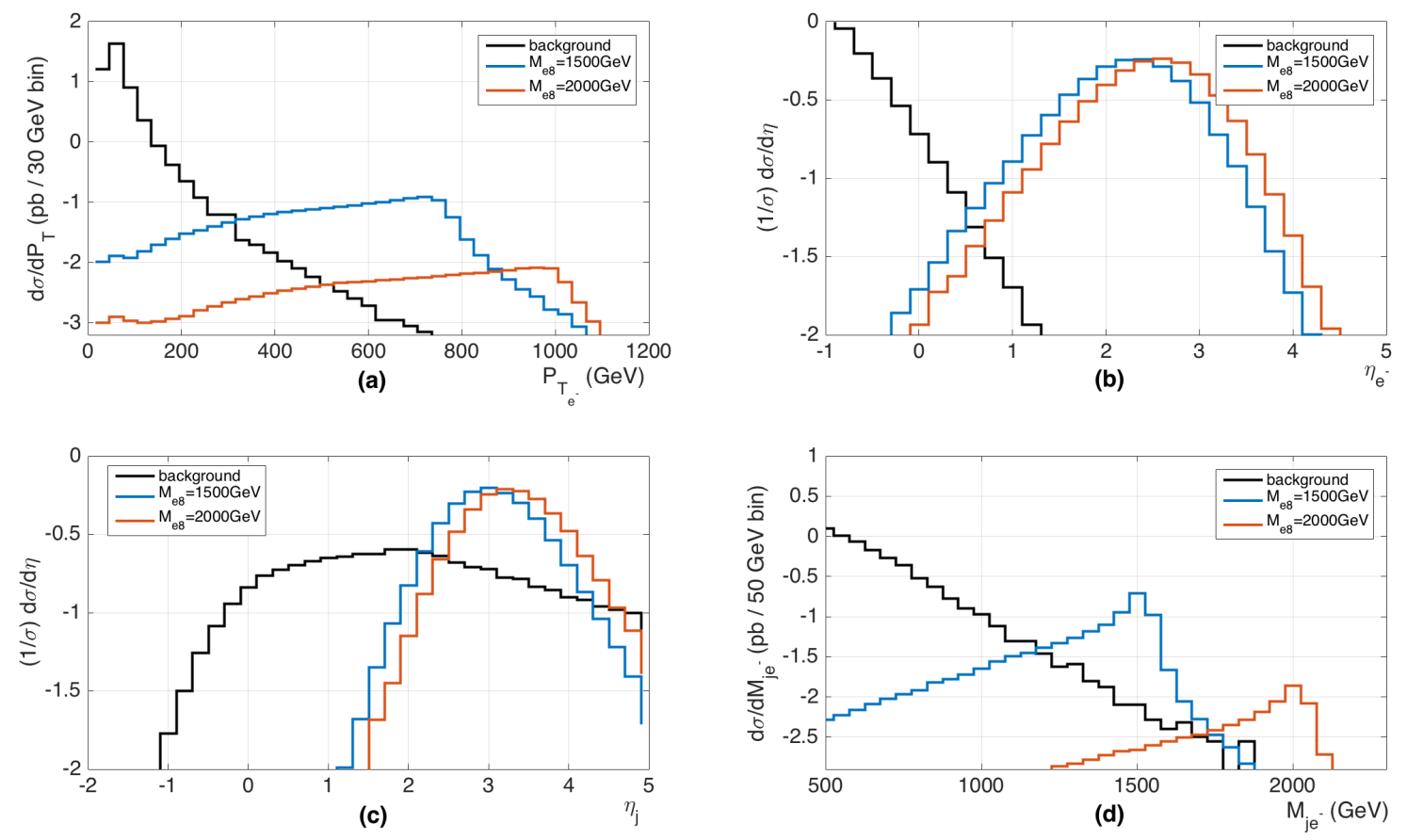

Figure 5. a) Transverse momentum distributions of final state jets (and electrons), b) pseudorapidity distributions of final state electrons, c) pseudorapidity distributions of final state jets and d) invariant mass distributions for signal and background at ERL60®FCC after generic cuts.

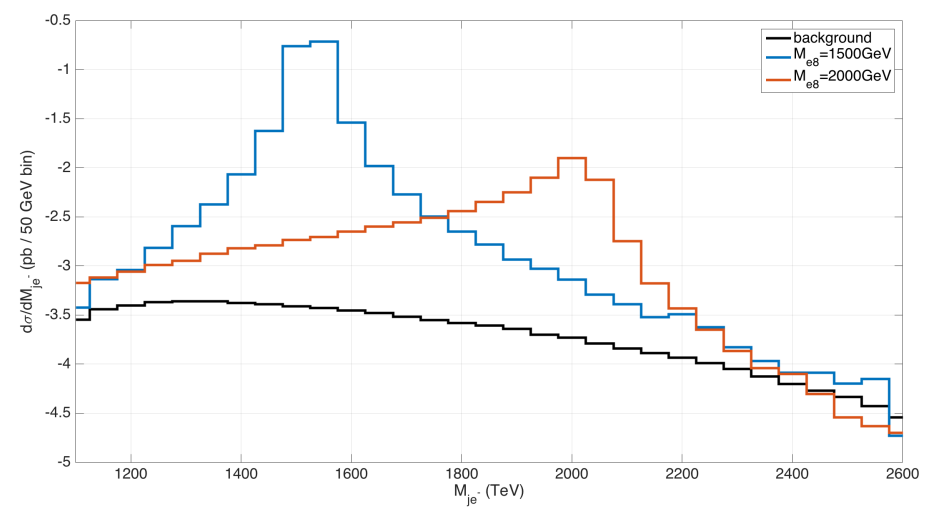

Figure 6. Invariant mass distributions for signal and background at ERL60®FCC after discovery cuts.

In order to determine discovery limits for color octet electron, we use following formula for statistical significance:

$$
S S=\sqrt{2[(S+B) \ln (1+(S / B))-S]}
$$

where $S$ and $B$ denote event numbers of signal and background, respectively. In addition to discovery cuts, mass window cuts are specified to determine $S$ and $B$ values as $M_{e_{8}}-2 \Gamma_{e_{8}}<M_{e j}<M_{e_{8}}+2 \Gamma_{e_{8}}$. Discovery $(S S=5)$ and observation $(S S=3)$ limits for $100 \mathrm{fb}^{-1}$ integrated luminosity are found to be 2900 and $3100 \mathrm{GeV}$, respectively.

Performing similar analysis for ILC $\otimes \mathrm{FCC}$ and assuming that $e_{8}$ is not observed by ERL60 $\otimes \mathrm{FCC}$ (that means $M_{e_{8}}>$ $3100 \mathrm{GeV}$ ) we determine following discovery cuts: $p_{T}>1500 \mathrm{GeV},-1.5<\eta_{e}<4.74$ and $0.5<\eta_{j}<4.74$. Invariant mass distributions after discovery cuts are presented in Fig. 7. Discovery limits for ILC $\otimes$ FCC with 10 and $100 \mathrm{fb}^{-1}$ 
Table III. Observation $(3 \sigma)$ and discovery $(5 \sigma)$ limits for $e_{8}$ at different ep colliders.

\begin{tabular}{|c|c|c|c|}
\hline \multirow{2}{*}{ Collider Name } & \multirow{2}{*}{$L_{\text {int }}, f^{-1}$} & \multicolumn{2}{|c|}{$M_{e_{8}}, \mathrm{TeV}$} \\
\cline { 3 - 4 } & & $3 \sigma$ & $5 \sigma$ \\
\hline \hline ERL60 $\otimes$ FCC & 100 & 3.1 & 2.9 \\
\hline \multirow{2}{*}{ ILC $\otimes$ FCC } & 10 & 8.4 & 8.1 \\
\cline { 2 - 4 } & 100 & 8.9 & 8.6 \\
\hline \multirow{2}{*}{ PWFA-LC $\otimes$ FCC } & 1 & 21.6 & 20.1 \\
\cline { 2 - 4 } & 10 & 24.3 & 23.1 \\
\hline
\end{tabular}

integrated luminosities are presented in Table III. Equation 5 and mass window $M_{e_{8}}-2 \Gamma_{e_{8}}<M_{e_{j}}<M_{e_{8}}+2 \Gamma_{e_{8}}$ have been used.

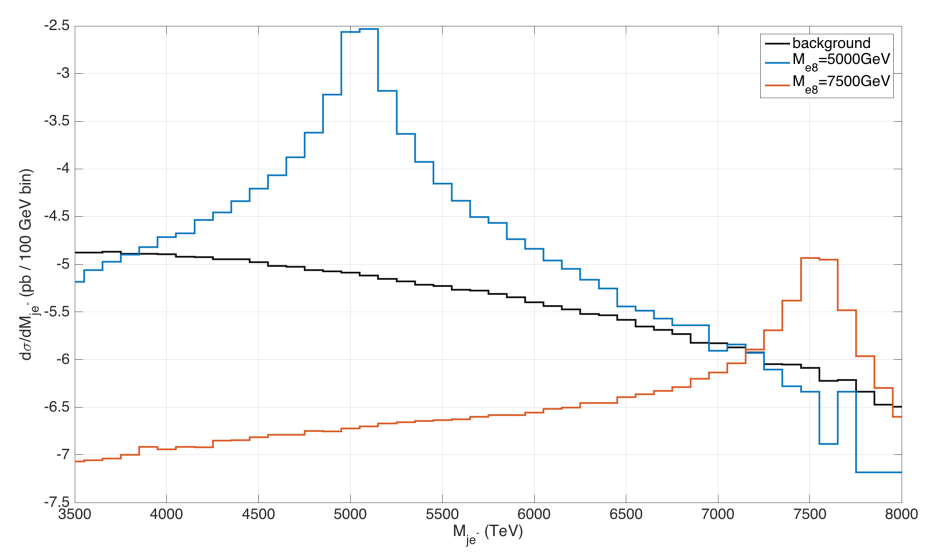

Figure 7. Invariant mass distributions for signal and background at ILC $\otimes \mathrm{FCC}$ after discovery cuts.

Similar consideration for PWFA-LC $\otimes$ FCC results in following discovery cuts: $p_{T}>4000 \mathrm{GeV}$ (assuming that $e_{8}$ is not observed at ILC $\otimes \mathrm{FCC}),-2.9<\eta_{e}<4.74$ and $-1.0<\eta_{j}<4.74$. Invariant mass distributions after these cuts are presented in Fig. 8. Discovery limits for PWFA-LC $\otimes$ FCC with 1 and $10 \mathrm{fb}^{-1}$ integrated luminosities are presented in last two rows of Table III.

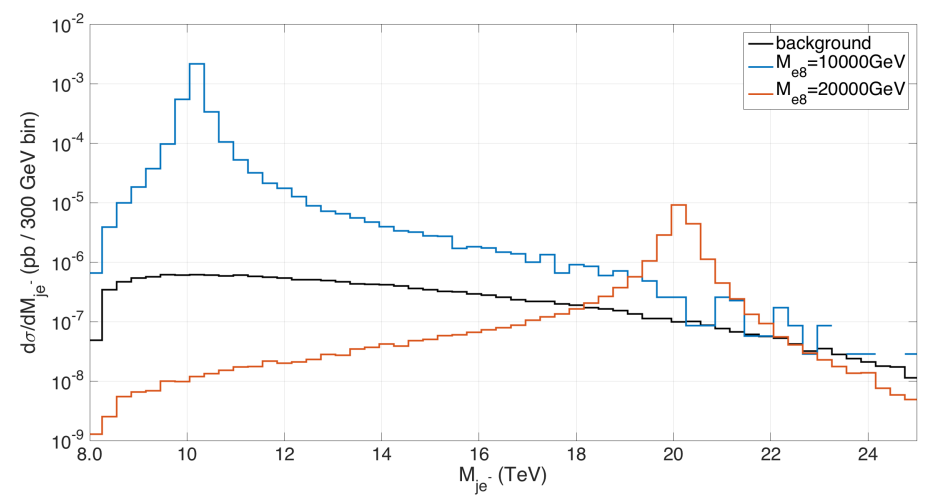

Figure 8. Invariant mass distributions for signal and background at PWFA-LC $\otimes$ FCC after discovery cuts.

\section{LIMITS ON COMPOSITENESS SCALE}

If the $e_{8}$ is discovered by FCC-pp option, ep colliders will give opportunity to estimate compositeness scale. In this regard, two distinct possibilities should be considered:

a) $e_{8}$ is discovered by FCC but not observed at e-FCC. In this case one can put lower limit on compositeness scale, 
b) $e_{8}$ is discovered by FCC and also observed at e-FCC. In this case one can determine compositeness scale.

In this section we present the analysis of these two possibilities for four different benchmark points, namely, $M_{e_{8}}=$ $2.5,5,7.5$ and $10 \mathrm{TeV}$.

A. $e_{8}$ is discovered by FCC but not observed at e-FCC

Since the $e_{8}$ mass is known one can determine optimal cuts for given $M_{e_{8}}$. Let us start by consideration of $M_{e_{8}}=2.5$ $\mathrm{TeV}$ at ILC $\otimes \mathrm{FCC}$. It is seen from Fig. 5 that $p_{T}>500 \mathrm{GeV},-1.30<\eta_{e}<4.74,0.50<\eta_{j}<3.00$ cuts drastically decrease the background whereas the signal is slightly affected. Similar analyses are performed for other collider options and $M_{e_{8}}$ values. Optimal cuts are presented in Table V. Invariant mass window $0.99 M e_{8}<M_{e j}<1.01 M e_{8}$ has been used in this particular analysis.

Table IV. Optimal cuts for determination of compositeness scale lower bounds.

\begin{tabular}{|c|c|c|c|c|c|c|c|c|c|}
\hline \multirow{2}{*}{ Collider } & \multirow{2}{*}{ Cut Type } & \multicolumn{2}{c|}{$M_{e_{8}}=2.5 \mathrm{TeV}$} & \multicolumn{2}{c|}{$M_{e_{8}}=5.0 \mathrm{TeV}$} & \multicolumn{2}{c|}{$M_{e_{8}}=7.5 \mathrm{TeV}$} & \multicolumn{2}{c|}{$M_{e_{8}}=10 \mathrm{TeV}$} \\
\cline { 2 - 10 } & & $\min$ & $\max$ & $\min$ & $\max$ & $\min$ & $\max$ & $\min$ & $\max$ \\
\hline \multirow{3}{*}{ ERL60 $\otimes \mathrm{FCC}$} & $\eta_{e}$ & 0.6 & 4.74 & - & - & - & - & - & - \\
\cline { 2 - 11 } & $\eta_{j}$ & 2.4 & 4.74 & - & - & - & - & - & - \\
\cline { 2 - 11 } & Mass Window & 2475 & 2525 & - & - & - & - & - & - \\
\hline \multirow{3}{*}{ ILC $\otimes \mathrm{FCC}$} & $\eta_{e}$ & -1.3 & 4.74 & -1.1 & 4.74 & -0.8 & 4.74 & - & - \\
\cline { 2 - 10 } & $\eta_{j}$ & 0.5 & 3.0 & 1.0 & 3.8 & 1.3 & 4.2 & - & - \\
\cline { 2 - 10 } & Mass Window & 2475 & 2525 & 4950 & 5050 & 7425 & 7575 & - & - \\
\hline \multirow{3}{*}{$\mathrm{PWFA-LC} \otimes \mathrm{FCC}$} & $\eta_{e}$ & -3.3 & 4.74 & -2.9 & 4.74 & -2.7 & 4.74 & -2.6 & 4.74 \\
\cline { 2 - 10 } & $\eta_{j}$ & -1.8 & 0.7 & -1.2 & 1.7 & -0.9 & 2.0 & -0.6 & 2.4 \\
\cline { 2 - 10 } & Mass Window & 2475 & 2525 & 4950 & 5050 & 7425 & 7575 & 9900 & 10100 \\
\hline
\end{tabular}

Applying cuts presented in Table $\mathrm{V}$ and $p_{T}>500 \mathrm{GeV}$ for all cases one can estimate achievable lower limits on compositeness scale. Using Eq. 5 we obtain $\Lambda$ values given in Table VI. As expected, lower bounds on compositeness scale is decreased with increasing value of the $e_{8}$ mass. It is seen that multi-hundred TeV lower bounds can be put on compositeness scale if $e_{8}$ is discovered at the FCC and not observed at ILC $\otimes$ FCC and PWFA-LC $\otimes F C C$.

Table V. Lower limits on compositeness scale in $\mathrm{TeV}$ units at the FCC based ep colliders

\begin{tabular}{|c|c|c|c|c|c|c|c|c|c|}
\hline \multirow{2}{*}{ Collider } & \multirow{2}{*}{$L_{i n t}, f b^{-1}$} & \multicolumn{2}{|c|}{$M_{e_{8}}=2.5 \mathrm{TeV}$} & \multicolumn{2}{|c|}{$M_{e_{8}}=5.0 \mathrm{TeV}$} & \multicolumn{2}{c|}{$M_{e_{8}}=7.5 \mathrm{TeV}$} & \multicolumn{2}{|c|}{$M_{e_{8}}=10 \mathrm{TeV}$} \\
\cline { 3 - 11 } & & $3 \sigma$ & $5 \sigma$ & $3 \sigma$ & $5 \sigma$ & $3 \sigma$ & $5 \sigma$ & $3 \sigma$ & $5 \sigma$ \\
\hline ERL60 $\otimes \mathrm{FCC}$ & 100 & 44 & 34 & - & - & - & - & - & - \\
\hline \multirow{2}{*}{ ILC $\otimes \mathrm{FCC}$} & 10 & 250 & 195 & 75 & 58 & 22 & 15 & - & - \\
& 100 & 450 & 350 & 135 & 105 & 42 & 32 & - & - \\
\hline \multirow{2}{*}{ PWFA-LC $\otimes \mathrm{FCC}$} & 1 & 220 & 170 & 200 & 150 & 190 & 145 & 110 & 80 \\
\cline { 2 - 10 } & 10 & 400 & 305 & 390 & 300 & 360 & 275 & 200 & 155 \\
\hline
\end{tabular}

\section{B. $e_{8}$ is discovered by FCC and observed at e-FCC}

In this case, the value of cross section at ep colliders which is inversely proportional to $\Lambda^{2}$ gives opportunity to determine compositeness scale directly. As an example, let us consider ILC $\otimes$ FCC case. In Fig. 9 we present $\Lambda$ dependence of $e_{8}$ production cross section for $M_{e_{8}}=2.5,5,7.5 \mathrm{TeV}$. Supposing that FCC discovers $e_{8}$ with 5 TeV mass and e-FCC measure cross section as $\sigma_{\text {exp }} \sim 2.50 \mathrm{fb}$, one can derive compositeness scale as $\Lambda_{\text {exp }}=100 \mathrm{TeV}$. 


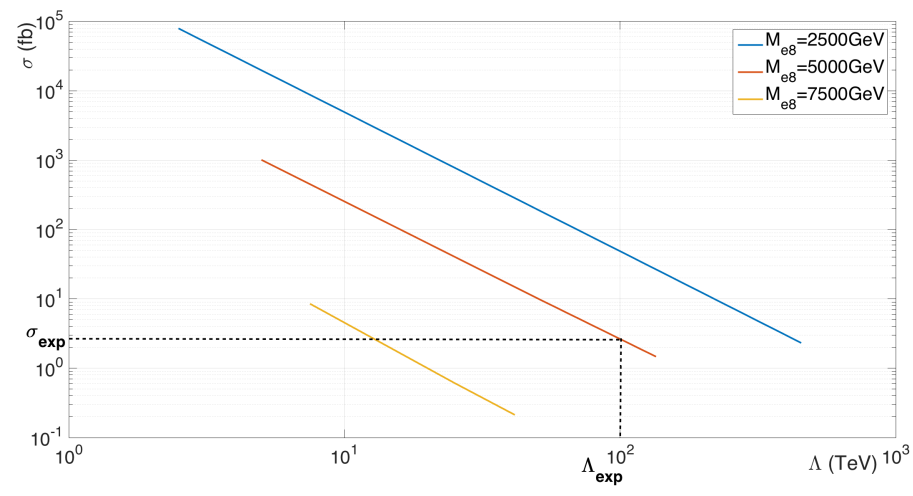

Figure 9. Cross section distributions with respect to compositeness scale for ILC $\otimes$ FCC collider.

\section{CONCLUSION}

It seems that FCC based ep colliders have great potential for $e_{8}$ searches. Discovery limits for $e_{8}$ at the LHC, FCC, ILC, PWFA-LC and FCC based ep colliders assuming $\Lambda=M e_{8}$ are summarized in Figure 10. Discovery limit $2.5 \mathrm{TeV}$ for LHC is taken from [6]. A discovery limit of $15 \mathrm{TeV}$ for FCC is obtained by rescaling LHC limit using the procedure developed by G. Salam and A. Weiler [35]. It is clear that discovery limits for pair production of $e_{8}$ at lepton colliders are approximately $\sqrt{s} / 2$. The search potential of ILC $\otimes$ FCC essentially exceeds that of LHC and linear colliders whereas is lower than FCC. Highest potential for $e_{8}$ search will be provided by PWFA-LC $\otimes$ FCC with discovery limit of $23 \mathrm{TeV}$ which is higher than $15 \mathrm{TeV}$ discovery limit provided by FCC pp collider. On the other hand, observation of $e_{8}$ at the FCC based ep colliders will provide an opportunity to determine compositeness scale, in some cases up to multi-hundred $\mathrm{TeV}$. In addition, polarized e-beams will give opportunity to clarify Lorentz structure of $e_{8}-e-g$ vertex (this subject is under consideration).

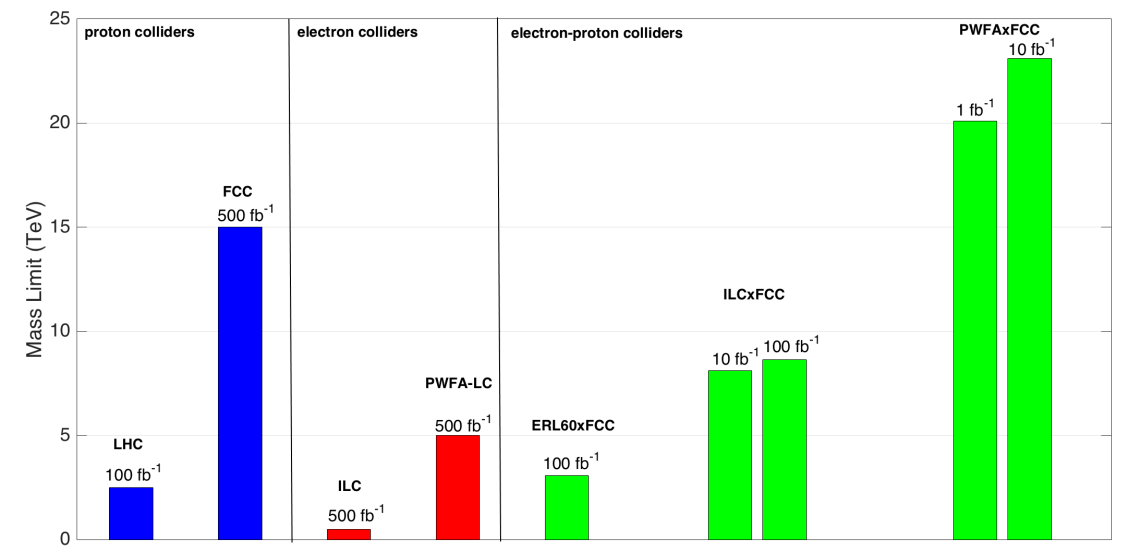

Figure 10. Discovery limits for $e_{8}$ at different pp, $e^{+} e^{-}$and ep colliders.

Finally, FCC based energy frontier ep colliders have great potential for BSM phenomena search, especially when related to the first SM family fermions. A similar statement is correct for FCC based $\mu$ p colliders if BSM phenomena are related to the second SM family fermions. Therefore, ERL60 $\otimes$ FCC should not be considered as the sole choise for the FCC based $\ell$ p colliders. Energy frontier $\ell$ p options should also be investigated at the same level. The proper choice for FCC based $\ell$ p collider option will be determined by the FCC results. 


\section{ACKNOWLEDGMENTS}

This study is supported by TUBITAK under the grant no 114F337. Authors are grateful to Gokhan Unel and Frank Zimmermann for useful discussions. Authors are also grateful to Subhadip Mitra and Tanumoy Mandal for sharing leptogluon MadGraph model file.

[1] ATLAS Collaboration, "Observation of a new particle in the search for the Standard Model Higgs boson with the ATLAS detector at the LHC", Phys. Lett. B 716 (2012) 1, doi:10.1016/j.physletb.2012.08.020, arXiv:1207.7214.

[2] CMS Collaboration, "Observation of a new boson at a mass of $125 \mathrm{GeV}$ with the CMS experiment at the LHC", Phys. Lett. B 716 (2012) 30, doi:10.1016/j.physletb.2012.08.021, arXiv:1207.7235

[3] J. C. Pati and A. Salam, Phys. Rev. D 10, 275 (1974) [Phys. Rev. D 11, 703 (1975)].

[4] I. A. D' Souza, C. S. Kalman, PREONS: Models of Leptons, Quarks and Gauge Bosons as Composite Objects, World Scientific Publishing Co. (1992).

[5] A. Celikel, M. Kantar and S. Sultansoy, "A search for sextet quarks and leptogluons at the LHC", Phys. Lett. B 443 (1998) 359.

[6] T. Mandal and S. Mitra, "Probing color octet electrons at the LHC", Phys. Rev. D 87 (2013) 095008.

[7] D. Gonçalves-Netto et al., "Looking for leptogluons", Phys. Rev. D 87 (2013) 094023.

[8] T. Jelinski and D. Zhuridov, "Leptogluons in dilepton production at LHC", e-Print arXiv:1510.04872 [hep-ph].

[9] T. Mandal, S. Mitra, and S. Seth. "Probing compositeness with the CMS eejj \& eej data" Physics Letters B 758 (2016): 219-225. arXiv:1602.01273v1

[10] A. Celikel and M. Kantar, "Resonance Production of New Resonances at ep and $\gamma$ p Colliders", Tr. J. of Physics 22 (1998) 401.

[11] M. Sahin, S. Sultansoy and S. Turkoz, "Resonant production of color octet electron at the LHeC", Phys. Lett. B 689 (2010) 172.

[12] M. Sahin, "Resonant production of spin-3/2 color octet electron at the LHeC", Acta Physica Polonica B 45 (2014) 1811.

[13] K. Cheung, "Muon-proton colliders: Leptoquarks, contact interactions and extra dimensions", AIP Conference Proceedings $542(2000) 160$.

[14] A. N. Akay, H. Karadeniz, M. Sahin and S. Sultansoy, "Indirect search for color octet electron at next-generation linear colliders", EPL 95 (2011) 31001.

[15] IceCube Collab. (M. G. Aartsen et al.), Phys. Rev. Lett. 113, 101101 (2014), arXiv:1405.5303 [astroph.HE].

[16] A. N. Akay et al., "New IceCube data and color octet neutrino interpretation of the PeV energy events", Int. J. Mod. Phys. A 30 (2015) 1550163.

[17] K. A. Olive et al. (Particle Data Group), Chin. Phys. C 38 (2014) 090001.

[18] F. Abe et al. (CDF Collaboration), "Search for Heavy Stable Charged Particles in 1.8-TeV pp(bar) collisions at the Fermilab Collider", Phys. Rev. Lett. 63 (1989) 1447. 15

[19] J. L. Hewett and T.G. Rizzo, "Much ado about leptoquarks: A comprehensive analysis", Phy. Rev. D 56 (1997) 5709.

[20] I. Abt et al. (H1 Collaboration), "A search for leptoquarks, leptogluons and excited leptons in H1 at HERA", Nucl. Phys. B 396 (1993) 3.

[21] T. Ahmed et al. (H1 Collaboration), "A search for leptoquarks and squarks at HERA", Z. Phys. C 64 (1994) 545.

[22] J. L. Abelleira Fernandez et al. (LHeC Study Group), "A Large Hadron Electron Collider at CERN: Report on the Physics and Design Concepts for Machine and Detector", J. Phys. G: Nucl. Part. Phys. 39, 075001 (2012).

[23] LHeC web page: http://lhec.web.cern.ch/erl-facility

[24] R. Hofstadter and R. W. McAllister, Phys. Rev. 98, 217 (1955).

[25] J. I. Friedman and H. W . Kendall, Ann . Rev . Nucl. Sci. 2, 203 (1972); See also the published versions of the Nobel lectures: R. E. Taylor, Rev. Mod. Phys. 6, 573 (1991); H. W. Kendall, Rev. Mod. Phys. 6, 597 (1991); J. I. Friedman, Rev. Mod. Phys. 6, 615 (1991).

[26] FCC web page: https://fcc.web.cern.ch.

[27] Y. C. Acar, U. Kaya, B. B. Oner and S. Sultansoy, "FCC based ep and $\mu$ p colliders", arXiv:1510.08284 [hep-ex].

[28] Y. C. Acar, U. Kaya, B. B. Oner and S. Sultansoy, "Main Parameters of LCxFCC Based Electron-Proton Colliders", arXiv:1602.03089 [hep-ex].

[29] S. Sultansoy, "FCC Based Lepton-Hadron and Photon-Hadron Colliders: Luminosity and Physics", FCC Week 2016, Rome, Italy.

[30] C. Adolphsen et al., The International Linear Collider Technical Design Report-Volume 3. II; arXiv:1306.6328

[31] J-P. Delahaye et al., A Beam Driven Plasma-Wakefield Linear Collider from Higgs Factory to Multi-TeV, in Proceedings of the Fifth International Particle Accelerator Conference, 2014 (Dresden, Germany), p. 3791.

[32] J. Alwall, M. Herquet, F. Maltoni, O. Mattelaer and T. Stelzer, JHEP 1106, 128 (2011); arXiv:1106.0522 [hep-ph].

[33] D. Stump et al., "Inclusive jet production, parton distributions and the search for new physics", JHEP 0310 (2003) 046.

[34] T. Sjostrand, S. Mrenna, and P. Z. Skands, "PYTHIA 6.4 physics and manual," JHEP 0605 (2006) 026, arXiv:hepph/0603175 [hep-ph].

[35] G. Salam and A. Weiler, "The Collider Reach project", http://collider-reach.web.cern.ch/collider-reach 